\title{
COMPACT EMBEDDED HYPERSURFACES WITH CONSTANT HIGHER ORDER ANISOTROPIC MEAN CURVATURES
}

\author{
YIJUN HE, HAIZHONG LI, HUI MA, AND JIANQUAN GE
}

\begin{abstract}
Given a positive function $F$ on $S^{n}$ which satisfies a convexity condition, for $1 \leq r \leq n$, we define the $r$-th anisotropic mean curvature function $H_{r}^{F}$ for hypersurfaces in $\mathbb{R}^{n+1}$ which is a generalization of the usual $r$-th mean curvature function. We prove that a compact embedded hypersurface without boundary in $\mathbb{R}^{n+1}$ with $H_{r}^{F}=$ constant is the Wulff shape, up to translations and homotheties. In case $r=1$, our result is the anisotropic version of Alexandrov Theorem, which gives an affirmative answer to an open problem of F. Morgan.
\end{abstract}

\section{INTRODUCTION}

Let $F: S^{n} \rightarrow \mathbb{R}^{+}$be a smooth function which satisfies the following convexity condition:

$$
\left(D^{2} F+F I\right)_{x}>0, \quad \forall x \in S^{n},
$$

where $S^{n}$ is the standard unit sphere in $\mathbb{R}^{n+1}, D^{2} F$ denotes the intrinsic Hessian of $F$ on $S^{n}$ and $I$ denotes the identity on $T_{x} S^{n},>0$ means that the matrix is positive definite. We consider the map

$$
\begin{aligned}
& \phi: S^{n} \rightarrow \mathbb{R}^{n+1}, \\
& x \rightarrow F(x) x+\left(\operatorname{grad}_{S^{n}} F\right)_{x},
\end{aligned}
$$

its image $W_{F}=\phi\left(S^{n}\right)$ is a smooth, convex hypersurface in $\mathbb{R}^{n+1}$ called the Wulff shape of $F$ (see [2], [3], [15], [10], [11], [12], [13], [17, [22, [23]). When $F \equiv 1$, the Wulff shape $W_{F}$ is just $S^{n}$.

Now let $X: M \rightarrow \mathbb{R}^{n+1}$ be a smooth immersion of a compact, orientable hypersurface without boundary. Let $\nu: M \rightarrow S^{n}$ denote its Gauss map.

2000 Mathematics Subject Classification. Primary 53C40; Secondary 53A10, 52A20.

Key words and phrases. Alexandrov Theorem, Wulff shape, embedded hypersurface, $r$-th anisotropic mean curvature.

The first author was partially supported by Youth Science Foundation of Shanxi Province, China (Grant No. 2006021001).

The second author was partially supported by the grant No. 10531090 of the NSFC and by SRFDP..

The third author was partially supported by NSFC grant No. 10501028 and NKBRPC No. $2006 \mathrm{CB} 805905$. 
Let $A_{F}=D^{2} F+F I, S_{F}=-\mathrm{d}(\phi \circ \nu)=-A_{F} \circ \mathrm{d} \nu . \quad S_{F}$ is called the $F$ Weingarten operator, and the eigenvalues of $S_{F}$ are called anisotropic principal curvatures. Let $\sigma_{r}$ be the elementary symmetric functions of the anisotropic principal curvatures $\lambda_{1}, \lambda_{2}, \cdots, \lambda_{n}$ :

$$
\sigma_{r}=\sum_{i_{1}<\cdots<i_{r}} \lambda_{i_{1}} \cdots \lambda_{i_{r}} \quad(1 \leq r \leq n) .
$$

We set $\sigma_{0}=1$. The $r$-th anisotropic mean curvature $H_{r}^{F}$ is defined by $H_{r}^{F}=\sigma_{r} / C_{n}^{r}$, also see Reilly [18]. $H^{F}=H_{1}^{F}$ is called the anisotropic mean curvature. If $F \equiv 1$, then $H_{r}^{F}=H_{r}$ is just the $r$-th mean curvature of hypersurfaces which has been studied by many authors (see [4], 14], [16], 21]). Thus, the $r$-th anisotropic mean curvature $H_{r}^{F}$ generalized the $r$-th mean curvature $H_{r}$ of hypersurfaces in the $(n+1)$-dimensional Euclidean space $\mathbb{R}^{n+1}$.

For hypersurfaces in $\mathbb{R}^{n+1}$, we have the following classical Alexandrov Theorem which was proved first by Alexandrov in [1] and later by Reilly in [19], Montiel-Ros in [16] and Hijazi-Montiel-Zhang in [8]:

Theorem 1.1. (Alexandrov Theorem) Let $X: M \rightarrow \mathbb{R}^{n+1}$ be a compact hypersurface without boundary embedded in Euclidean space. If $H=$ constant, then $X(M)$ is a sphere.

Following from a modification of Reilly's proof, Ros showed in [20] that the sphere is the only compact embedded hypersurface without boundary with constant scalar curvature in $\mathbb{R}^{n+1}$, which gave a partial answer to Yau's conjecture 24]. Thereafter, Ros [21] extended his result to any $r$-th mean curvature, and later, Montiel and Ros gave another proof in [16]. Explicitly, they proved:

Theorem 1.2. ([16], [21]) Let $X: M \rightarrow \mathbb{R}^{n+1}$ be a compact hypersurface without boundary embedded in Euclidean space. If $H_{r}=$ constant for some $r=1, \cdots, n$, then $X(M)$ is a sphere.

In this paper, we prove the following anisotropic version of Theorem 1.2.

Theorem 1.3. Let $X: M \rightarrow \mathbb{R}^{n+1}$ be a compact hypersurface without boundary embedded in Euclidean space. If $H_{r}^{F}=$ constant for some $r=1, \cdots, n$, then up to translations, $X(M)=\rho W_{F}$, where $\rho=-1 / H_{1}^{F}$ is a constant.

Remark 1.1. For $n=1$, Morgan [15] proved that Theorem 1.3 still holds for a more general condition: $F$ is only a continuous norm on $\mathbb{R}^{2}$ and $X: M \rightarrow \mathbb{R}^{2}$ is a closed curve immersed in $\mathbb{R}^{2}$. In case $r=1$, Theorem 1.3 is actually the anisotropic version of Alexandrov Theorem, which gives an affirmative answer to the following open problem proposed by Morgan in the same paper: Whether an embedded equilibrium, i.e. hypersurfaces with constant anisotropic mean curvature in Euclidean space, must be the Wulff shape? We also note that M. Koiso stated this conjecture in [9]. 
Remark 1.2. Theorem 1.2 follows by choosing $F \equiv 1$ in Theorem 1.3

\section{Preliminaries}

Let $X: M \rightarrow \mathbb{R}^{n+1}$ be a compact connected hypersurface immersed in Euclidean space. Let $\nu: M \rightarrow S^{n}$ denote its Gauss map. Suppose there exists a point where all the principal curvatures with respect to $\nu$ are positive. By the positiveness of $A_{F}$, all the anisotropic principal curvatures are positive at this point. Using the results of Gårding ([5]), we have the following lemma (cf. Montiel-Ros [16]):

Lemma 2.1. Let $X: M \rightarrow \mathbb{R}^{n+1}$ be a compact connected hypersurface without boundary. Suppose that there exists a point where all the principal curvatures are positive. Assume $H_{r}^{F}>0$ holds on every point of $M$, then the same holds for $H_{k}^{F}, k=1, \cdots, r-1$. Moreover

$$
\left(H_{k}^{F}\right)^{(k-1) / k} \leq H_{k-1}^{F}, \quad\left(H_{k}^{F}\right)^{1 / k} \leq H_{1}^{F}, \quad k=1, \cdots, r .
$$

If $k \geq 2$, the equality in the above inequalities happens only at points where all the anisotropic principal curvatures are equal.

Let $\left\{e_{1}, \cdots, e_{n}\right\}$ be a local orthogonal frame of $X: M \rightarrow \mathbb{R}^{n+1}$, then we have the structure equations:

$$
\left\{\begin{array}{l}
\mathrm{d} X=\sum_{i} \omega_{i} e_{i} \\
\mathrm{~d} \nu=-\sum_{i j} h_{i j} \omega_{j} e_{i} \\
\mathrm{~d} e_{i}=\sum_{j} \omega_{i j} e_{j}+\sum_{j} h_{i j} \omega_{j} \nu \\
\mathrm{d} \omega_{i}=\sum_{j} \omega_{i j} \wedge \omega_{j} \\
\mathrm{~d} \omega_{i j}-\sum_{k} \omega_{i k} \wedge \omega_{k j}=-\frac{1}{2} \sum_{k l} R_{i j k l} \theta_{k} \wedge \theta_{l}
\end{array}\right.
$$

where $\omega_{i j}+\omega_{j i}=0, R_{i j k l}+R_{i j l k}=0$, and $R_{i j k l}$ are the components of the Riemannian curvature tensor of $M$ with respect to the induced metric $\mathrm{d} X \cdot \mathrm{d} X$.

Let $s_{i j}$ denote the coefficient of $S_{F}$ with respect to $\left\{e_{1}, \cdots, e_{n}\right\}$, that is

$$
-\mathrm{d}(\phi \circ \nu)=-A_{F} \circ \mathrm{d} \nu=\sum_{i, j} s_{i j} \omega_{j} e_{i},
$$

where $\phi$ is defined in (2).

We call the eigenvalues of $S_{F}$ to be anisotropic principal curvatures, and denote them by $\lambda_{1}, \cdots, \lambda_{n}$. From the positive definiteness of $A_{F}$, there exists a non-singular matrix $C$ such that $A_{F}=C^{T} C$, so $S_{F}=-A_{F} \circ \mathrm{d} \nu$ is similar to the real symmetric matrix $-C \circ \mathrm{d} \nu \circ C^{T}$. Thus, the anisotropic principal curvatures are all real. Moreover, if $\lambda_{1}=\cdots=\lambda_{n}$, we have $S_{F}=H_{1}^{F} I$, so $-\mathrm{d}(\phi \circ \nu)=H_{1}^{F} \mathrm{~d} X$ by (44) and (5). Thus, we have the following lemma (cf. [6], 7], [17]): 
Lemma 2.2. Let $X: M \rightarrow \mathbb{R}^{n+1}$ be a compact hypersurface without boundary. If $\lambda_{1}=\lambda_{2}=\cdots=\lambda_{n}=$ const $\neq 0$, then up to translations, $X(M)=\rho W_{F}$, where $\rho=-1 / H_{1}^{F}$.

We define $s_{i j k}$ by

$$
\mathrm{d} s_{i j}+\sum_{k} s_{i k} \omega_{k j}+\sum_{k} s_{k j} \omega_{k i}=\sum_{k} s_{i j k} \omega_{k} .
$$

Taking exterior differentiation of (5) and using (44), we get

$$
s_{i j k}=s_{i k j}
$$

Lemma 2.3. Let $X: M \rightarrow \mathbb{R}^{n+1}$ be a compact hypersurface without boundary. If $n \geq 2$ and $\lambda_{1}=\lambda_{2}=\cdots=\lambda_{n} \neq 0$, then $\lambda_{1}=\lambda_{2}=\cdots=\lambda_{n}=$ const, so up to translations, $X(M)=\rho W_{F}$, where $\rho=-1 / H_{1}^{F}$.

Proof. From (7) and $s_{j i}=H_{1}^{F} \delta_{i j}$, we have

$$
e_{i}\left(H_{1}^{F}\right)=\sum_{j} s_{j i j}=\sum_{j} s_{j j i}=n e_{i}\left(H_{1}^{F}\right), \quad 1 \leq i \leq n .
$$

Therefore $\lambda_{1}=\lambda_{2}=\cdots=\lambda_{n}=H_{1}^{F}$ is a constant, then the conclusion follows from Lemma 2.2.

We define $F^{*}: \mathbb{R}^{n+1} \rightarrow \mathbb{R}$ to be (see [2]):

$$
F^{*}(x)=\sup \left\{\frac{\langle x, z\rangle}{F(z)} \mid z \in S^{n}\right\}
$$

Proposition 2.1. Let $x \in \mathbb{R}^{n+1} \backslash\{0\}, y, z \in S^{n}$, then we have:

(i) $\langle\phi(y), z\rangle \leq F(z)$, and the equality holds if and only if $y=z$;

(ii) $\langle x, y\rangle \leq F^{*}(x) F(y)$, and the equality holds if and only if $x=F^{*}(x) \phi(y)$.

Proof. Proof of (i). It is obvious that $\langle\phi(y), z\rangle \leq F(z)$ is equivalent to $\langle\phi(y)-\phi(z), z\rangle \leq$ 0 . The function $\Phi: S^{n} \times S^{n} \rightarrow \mathbb{R}$ defined by

$$
\Phi(y, z)=\langle\phi(y)-\phi(z), z\rangle
$$

is smooth, so it attained its maximum at some point $\left(y_{0}, z_{0}\right)$ because $S^{n} \times S^{n}$ is compact. By differentiating the function $\Phi(y, z)$ with respect to $y$ at the point $\left(y_{0}, z_{0}\right)$, we get

$$
\left\langle A_{F} \circ \mathrm{d} y, z\right\rangle_{\left(y_{0}, z_{0}\right)}=0 .
$$

Thus, from the positiveness of $A_{F}, z_{0}$ is orthogonal to $S^{n}$ at the point $y_{0}$, so, we must have $y_{0}= \pm z_{0}$. Notice that $\Phi\left(z_{0}, z_{0}\right)=0, \Phi\left(-z_{0}, z_{0}\right)=-F\left(z_{0}\right)-F\left(-z_{0}\right)<0$, the function $\Phi$ must attain its maximum 0 at the point $\left(z_{0}, z_{0}\right)$, so $\langle\phi(y), z\rangle \leq F(z)$. If $\langle\phi(y), z\rangle=F(z)$, then $\Phi$ obtains its maximum 0 at the point $(y, z)$, by the same reason we have $y=z$. 
Proof of (ii). It is obvious that $\langle x, y\rangle \leq F^{*}(x) F(y)$ by the definition of $F^{*}$. Now we suppose that $\langle x, y\rangle=F^{*}(x) F(y)$, then the function $\left\langle x-F^{*}(x) \phi(y), y\right\rangle$ obtains its maximum 0 at the point $(x, y)$. So, differentiating it with respect to $y$, we get

$$
\left\langle x-F^{*}(x) \phi(y), \mathrm{d} y\right\rangle=0 .
$$

Thus, it follows that $x-F^{*}(x) \phi(y)$ is orthogonal to $S^{n}$ at $y$, that is, $x-F^{*}(x) \phi(y)=k y$ for some $k$. Then from $\left\langle x-F^{*}(x) \phi(y), y\right\rangle=0$, we have $x-F^{*}(x) \phi(y)=0$.

Proposition 2.2. We have:

(i) $F^{*}(x)>0, \forall x \in \mathbb{R}^{n+1} \backslash\{0\}$;

(ii) $F^{*}(t x)=t F^{*}(x), \forall x \in \mathbb{R}^{n+1}, t>0$;

(iii) $F^{*}(x+y) \leq F^{*}(x)+F^{*}(y), \forall x, y \in \mathbb{R}^{n+1}$, and the equality holds if and only if $x=0$, or $y=0$ or $x=k y$ for some $k>0$.

(iv) $W_{F}=\left\{x \in \mathbb{R}^{n+1} \mid F^{*}(x)=1\right\}$.

Proof. (i) and (ii) follow from the definition of $F^{*}$. By the definition of $F^{*}$ and (ii) of Proposition 2.1, we easily get (iv). We now prove (iii). Suppose $x, y \neq 0$. Let $z \in S^{n}$ be such that $F^{*}(x+y)=\langle x+y, z\rangle / F(z)$, then we have

$$
F^{*}(x+y)=\langle x+y, z\rangle / F(z)=\langle x, z\rangle / F(z)+\langle y, z\rangle / F(z) \leq F^{*}(x)+F^{*}(y),
$$

with the equality holding if and only if $F^{*}(x)=\langle x, z\rangle / F(z)$ and $F^{*}(y)=\langle y, z\rangle / F(z)$. So, if the equality holds, then from (ii) of Proposition 2.1 we have

$$
x=F^{*}(x) \phi(z), \quad y=F^{*}(y) \phi(z) .
$$

Thus, $x=F^{*}(x) / F^{*}(y) y$.

From Proposition 2.2, for any $x \in \mathbb{R}^{n+1} \backslash\{0\}$, we have $x / F^{*}(x) \in W_{F}$, thus there exists a unique $\psi(x) \in S^{n}$ such that $x=F^{*}(x) \phi(\psi(x))$. From the implicit function theorem and the convexity of $F$, the function $F^{*}: \mathbb{R}^{n+1} \backslash\{0\} \rightarrow \mathbb{R}^{+}$and $\psi: \mathbb{R}^{n+1} \backslash\{0\} \rightarrow S^{n}$ are smooth.

\section{F-FOCAL POINT AND $F$-CUT POINT}

We define $d_{F}: \mathbb{R}^{n+1} \times \mathbb{R}^{n+1} \rightarrow \mathbb{R}$ to be $d_{F}(x, y)=F^{*}(y-x)$, then we have $d_{F}(x, y)>0$ when $x \neq y, d_{F}(x, x)=0$ and $d_{F}(x, z) \leq d_{F}(x, y)+d_{F}(y, z)$. Note that in general $d_{F}(x, y) \neq d_{F}(y, x)$; and when $F \equiv 1, d_{F}$ is just the Euclidean distance function $d$.

For every $p \in \mathbb{R}^{n+1}$, let $\exp _{p}$ be the exponential map in $\mathbb{R}^{n+1}$ at the point $p$, then $\exp _{p}(u)=p+u$. So, from the definition of $d_{F}$, we have

$$
d_{F}\left(p, \exp _{p}(t \phi(Y))\right)=t \text {, for every } Y \in S^{n} \text { and } t \in \mathbb{R}^{+} .
$$


Now, let $X: M \rightarrow \mathbb{R}^{n+1}$ be a compact embedded hypersurface without boundary, and $\nu$ be the unit inner normal vector field of $M$. For convenience, we identify each point $p \in M$ with its image $X(p) \in \mathbb{R}^{n+1}$.

For each $y \in \mathbb{R}^{n+1}$, define

$$
d_{F}(M, y)=\inf \left\{d_{F}(p, y) \mid p \in M\right\} .
$$

We define a function $c: M \rightarrow \mathbb{R}^{+}$to be such that $c(p)$ is the greatest $t \in(0, \infty)$ satisfying $d_{F}\left(M, \exp _{p}(t \phi \circ(\nu(p)))\right)=t$. We call $\exp _{p}(c(p) \phi \circ \nu(p))$ the $F$-cut point of $p \in M$.

For $p \in M$, let $\gamma_{p}$ be the ray $\gamma_{p}:[0, \infty) \rightarrow \mathbb{R}^{n+1}$ defined by:

$$
\gamma_{p}(t)=p+t \phi \circ \nu(p), \quad \forall t \in[0, \infty),
$$

and $\Gamma_{p}=\gamma_{p}([0, c(p)))$. Then we have

Lemma 3.1. For $p, q \in M, p \neq q$, we have $\Gamma_{p} \cap \Gamma_{q}=\emptyset$.

Proof. Suppose $x \in \Gamma_{p} \cap \Gamma_{q}$, then there exists $0<t<\min (c(p), c(q))$ such that

$$
x=\exp _{p}(t \phi \circ \nu(p))=\exp _{q}(t \phi \circ \nu(q)),
$$

by the definition of $c(p), c(q)$ and (9).

Suppose $t<s<c(p)$, then from (iii) of Proposition 2.2, we have

$$
\begin{aligned}
d_{F}\left(q, \exp _{p}(s \phi \circ \nu(p))\right) & <d_{F}(q, x)+d_{F}\left(x, \exp _{p}(s \phi \circ \nu(p))\right) \\
& =d_{F}(p, x)+d_{F}\left(x, \exp _{p}(s \phi \circ \nu(p))\right) \\
& =d_{F}\left(p, \exp _{p}(s \phi \circ \nu(p))\right) \\
& =d_{F}\left(M, \exp _{p}(s \phi \circ \nu(p))\right),
\end{aligned}
$$

a contradiction.

Consider the map: $\Psi: M \times \mathbb{R} \rightarrow \mathbb{R}^{n+1}, \Psi(p, t)=\exp _{p}(t \phi \circ \nu(p))$. If $(p, t)$ is a critical point of the map $\Psi$, then we call $\exp _{p}(t \phi \circ \nu(p))$ an $F$-focal point of $p \in M$. Because $\exp _{p}(t \phi \circ \nu(p))=p+t \phi \circ \nu(p)$, so through direct computation, we have

$$
\mathrm{d}\left(\exp _{p}(t \phi \circ \nu(p))\right)=\left(I-t S_{F}\right) \circ \mathrm{d} p+(\phi \circ \nu(p)) \mathrm{d} t .
$$

From (11), $\exp _{p}(t \phi \circ \nu(p))$ is an $F$-focal point of $p$ if and only if the matrix $I-t S_{F}$ is degenerate. So, the first $F$-focal point of $p$ along the ray $\gamma_{p}$ is $\exp _{p}\left(1 / \lambda_{\max } \phi \circ \nu(p)\right)$, where $\lambda_{\max }$ is the greatest positive anisotropic principal curvature at $p$.

Remark 3.1. When $F=1, F$-cut point, $F$-focal point is the cut point and the focal point of hypersurfaces in the Euclidean space respectively.

Lemma 3.2. Either $(p, c(p))$ is a critical point of the map $\Psi$, or there exists at least one point $q \in M, q \neq p$, such that $d_{F}\left(q, \exp _{p}(c(p) \phi \circ \nu(p))\right)=c(p)$. 
Proof. We choose $\varepsilon_{i}>0$, such that $\lim _{i \rightarrow \infty} \varepsilon_{i}=0$. Let $a_{i}=\exp _{p}\left(\left(c(p)+\varepsilon_{i}\right) \phi \circ \nu(p)\right)$, $a=\exp _{p}(c(p) \phi \circ \nu(p))$. The continuity of $\Psi$ implies that $\lim _{i \rightarrow \infty} a_{i}=a$. From the definition of $c(p)$, there exists points $q_{i} \in M$, such that $d_{F}\left(q_{i}, a_{i}\right)=d_{F}\left(M, a_{i}\right)=$ $c(p)+\varepsilon_{i}^{\prime}$, where $\varepsilon_{i}^{\prime}<\varepsilon_{i}$, possibly $<0$. From the compactness of $M$, there exists a convergent subsequence of $\left\{q_{i}\right\}$, again denoted by $\left\{q_{i}\right\}$ such that $\lim _{i \rightarrow \infty} q_{i}=q$. Then we divided into two cases:

Case 1. $q \neq p$. In this case we have

$$
\lim _{i \rightarrow \infty} d_{F}\left(q_{i}, a_{i}\right)=d_{F}(q, a),
$$

and

$$
\lim _{i \rightarrow \infty} d_{F}\left(M, a_{i}\right)=d_{F}(M, a)=c(p) .
$$

So, we have $d_{F}(q, a)=c(p)$, as expected.

Case 2. $q=p$. Suppose $(p, c(p))$ is not a critical point of the map $\Psi$, then there exists a neighborhood $U$ of $(p, c(p)) \in M \times \mathbb{R}$ such that $\left.\Psi\right|_{U}: U \rightarrow \Psi(U)$ is a diffeomorphism. And we have $\lim _{i \rightarrow \infty} d_{F}\left(q_{i}, a_{i}\right)=d_{F}(q, a)=c(p)$, so $\lim _{i \rightarrow \infty} \varepsilon_{i}^{\prime}=0$. Therefore, for a sufficient large $i$, we have $\left(p, c(p)+\varepsilon_{i}\right),\left(q_{i}, c(p)+\varepsilon_{i}^{\prime}\right) \in U$. But

$$
\exp _{p}\left(\left(c(p)+\varepsilon_{i}\right) \phi \circ \nu(p)\right)=\exp _{q_{i}}\left(\left(c(p)+\varepsilon_{i}^{\prime}\right) \phi \circ \nu\left(q_{i}\right)\right),
$$

thus we have $p=q_{i}$ and $\varepsilon_{i}=\varepsilon_{i}^{\prime}$, a contradiction.

Lemma 3.3. $c(p) \leq 1 / \lambda_{\max }$, where $\lambda_{\max }$ is the greatest positive anisotropic principal curvature at $p$.

Proof. Let $t>1 / \lambda_{\max }$. We define a function $h: M \rightarrow \mathbb{R}$ by

$$
h(q)=F^{*}(p+t \phi \circ \nu(p)-q), \forall q \in M .
$$

We prove that $p$ is not a local minimum point, so $t>c(p)$, thus the conclusion follows.

Because, $(p+t \phi \circ \nu(p)-q) / h(q) \in W_{F}$, we can define a function $Y: M \rightarrow S^{n}$ by

$$
p+t \phi \circ \nu(p)-q=h(q) \phi \circ Y(q),
$$

and we have $Y(p)=\nu(p)$.

Let $\gamma:(-\varepsilon, \varepsilon) \rightarrow M$ be a smooth curve such that $\gamma(0)=p$, we denote $h(s)=$ $h(\gamma(s)), \gamma(s)=X(\gamma(s)), Y(s)=Y(\gamma(s)), \nu(s)=\nu(\gamma(s))$ for simplicity. Then, we have

$$
p+t \phi \circ \nu(p)-\gamma(s)=h(s) \phi \circ Y(s) .
$$

Differentiating (14), we get

$$
h^{\prime}(s) \cdot \phi(Y(s))+h(s) \cdot(\phi(Y))^{\prime}(s)=-\gamma^{\prime}(s) .
$$

From $\langle Y, Y\rangle=1$, we have $\left\langle Y^{\prime}(s), Y(s)\right\rangle=0$, together with $(\phi(Y))^{\prime}(s)=A_{F} \circ Y^{\prime}(s)$ we have

$$
\left\langle(\phi(Y))^{\prime}(s), Y(s)\right\rangle=0 .
$$


Thus, by taking inner product with $Y(s)$ in (15), we get

$$
h^{\prime}(s) F(Y(s))=-\left\langle\gamma^{\prime}(s), Y(s)\right\rangle .
$$

From (17) and $Y(0)=\nu(p)$, we have $h^{\prime}(0)=-\left\langle\gamma^{\prime}(0), \nu(p)\right\rangle / F(\nu(p))=0$, so $p$ is a extreme point of the function $h$. And we have

$$
t(\phi(Y))^{\prime}(0)=t A_{F} \circ Y^{\prime}(0)=-\gamma^{\prime}(0),
$$

Differentiating (15), we get

$$
h^{\prime \prime}(s) \cdot \phi(Y(s))+2 h^{\prime}(s) \cdot(\phi(Y))^{\prime}(s)+h(s)(\phi(Y))^{\prime \prime}(s)=-\gamma^{\prime \prime}(s) .
$$

Differentiating (16), we get

$$
\left\langle(\phi(Y))^{\prime \prime}(s), Y(s)\right\rangle=-\left\langle(\phi(Y))^{\prime}(s), Y^{\prime}(s)\right\rangle
$$

Thus, by taking inner product with $Y(s)$ in (19) and using (16), (20), we get

$$
h^{\prime \prime}(s) \cdot F(Y(s))-h(s)\left\langle A_{F} \circ Y^{\prime}(s), Y^{\prime}(s)\right\rangle=-\left\langle\gamma^{\prime \prime}(s), Y(s)\right\rangle .
$$

Evaluating (21) at $s=0$, using $Y(0)=\nu(p)$ and (18) we have

$$
h^{\prime \prime}(0) \cdot F(\nu(p))-\frac{1}{t}\left\langle A_{F}^{-1} \circ \gamma^{\prime}(0), \gamma^{\prime}(0)\right\rangle=-\left\langle\gamma^{\prime \prime}(0), \nu(p)\right\rangle=\left\langle\gamma^{\prime}(0), \nu^{\prime}(0)\right\rangle .
$$

Now, let $\gamma$ be such a curve that satisfies $-A_{F} \circ \nu^{\prime}(0)=\lambda_{\max } \gamma^{\prime}(0)$, that is, $\gamma^{\prime}(0)$ is the eigenvector corresponding to the maximum positive eigenvalue of $S_{F}=-A_{F} \circ \mathrm{d} \nu$. Then, we have

$$
h^{\prime \prime}(0)=\frac{\lambda_{\max }}{t F(\nu(p))}\left(\frac{1}{\lambda_{\max }}-t\right)\left\langle A_{F}^{-1} \circ \gamma^{\prime}(0), \gamma^{\prime}(0)\right\rangle<0,
$$

because $A_{F}^{-1}$ is positive definite.

So, $p$ is not a local minimum point of the function $h$.

Lemma 3.4. The map $c: M \rightarrow \mathbb{R}^{+}$is continuous.

Proof. Let $p_{i} \in M$ be such that $\lim _{i \rightarrow \infty} p_{i}=p$, we need to prove $\lim _{i \rightarrow \infty} c\left(p_{i}\right)=c(p)$. For any $q \in M$, we have

$d\left(q, \exp _{q}(c(q) \phi(\nu(q)))\right)=c(q) \sqrt{\left|\left(\operatorname{grad}_{S^{n}} F\right)(\nu(q))\right|^{2}+[F(\nu(q))]^{2}}<$ the diameter of $M$, so the function $c$ is bounded.

Firstly, we prove $\limsup _{i \rightarrow \infty} c\left(p_{i}\right) \leq c(p)$. For any $\varepsilon>0$, there do not exist infinitely many indices $i$ such that $c\left(p_{i}\right)>c(p)+\varepsilon$. Otherwise, by the definition of $c\left(p_{i}\right)$, we have

$$
d_{F}\left(p_{i}, \exp _{p}\left((c(p)+\varepsilon) \phi\left(\nu\left(p_{i}\right)\right)\right)\right)=c(p)+\varepsilon,
$$

and, by the continuity of the function $d_{F}, d_{F}\left(p, \exp _{p}((c(p)+\varepsilon) \phi(\nu(p)))\right)=c(p)+\varepsilon$, which contradicts the definition of $c(p)$. Therefore $\limsup _{i \rightarrow \infty} c\left(p_{i}\right) \leq c(p)+\varepsilon$, for any $\varepsilon>0$, which proves the claim. 
Secondly, we prove $\liminf _{i \rightarrow \infty} c\left(p_{i}\right) \geq c(p)$. Let $\bar{t}=\liminf _{i \rightarrow \infty} c\left(p_{i}\right)$. Consider a subsequence of $\left\{c\left(p_{i}\right)\right\}$, again denoted by $c\left(p_{i}\right)$, which converges to $\bar{t}$. It is obvious an accumulation point of $F$-focal points is $F$-focal point, if for any such subsequence, the points $\exp _{p_{i}}\left(c\left(p_{i}\right) \phi\left(\nu\left(p_{i}\right)\right)\right)$ are $F$-focal points of $p_{i}$, then $\exp _{p}(c(p) \phi(\nu(p)))$ is an $F$-focal point of $p$, hence $\bar{t} \geq c(p)$ by Lemma 3.3 .

Suppose, therefore, there exists a subsequence of $c\left(p_{i}\right)$ (again denoted by $c\left(p_{i}\right)$ ), such that $\exp _{p_{i}}\left(c\left(p_{i}\right) \phi\left(\nu\left(p_{i}\right)\right)\right)$ is not $F$-focal point of $p_{i}$. By Lemma 3.2, there exists $q_{i} \in$ $M$ such that $d_{F}\left(q_{i}, \exp _{p_{i}}\left(c\left(p_{i}\right) \phi\left(\nu\left(p_{i}\right)\right)\right)\right)=c\left(p_{i}\right)$. Taking, if necessary, a subsequence, we may suppose that $\lim _{i \rightarrow \infty} q_{i}=q \in M$. If $p \neq q$, by taking limit we see that, $d_{F}\left(q, \exp _{p}(\bar{t} \phi(\nu(p)))\right)=d_{F}\left(p, \exp _{p}(\bar{t} \phi(\nu(p)))\right)$, hence $\bar{t} \geq c(p)$. If $p=q$, then for any neighborhood $V=U \times(\bar{t}-\varepsilon, \bar{t}+\varepsilon)$ of $(p, \bar{t})$, there exists $i$, such that $p_{i}, q_{i} \in U$ and $c\left(p_{i}\right) \in(\bar{t}-\varepsilon, \bar{t}+\varepsilon)$. Choose $\bar{t}-\varepsilon<s<c\left(p_{i}\right)$, then we have $\exp _{p_{i}}\left(s \phi\left(\nu\left(p_{i}\right)\right)\right) \neq$ $\exp _{q_{i}}\left(s \phi\left(\nu\left(q_{i}\right)\right)\right)$ by Lemma 3.1, so the map $\left.\Psi\right|_{V}: V \rightarrow \Psi(V)$ can not be injective. Thus, $(p, \bar{t})$ is a critical point of $\Psi$.

\section{AN INTEGRAL INEQUALITY OF COMPACT HYPERSURFACES}

In this section we derive an integral inequality of compact hypersurface without boundary embedded in Euclidean space (Theorem 4.2) which plays an important role in the proof of our main theorem. First, we recall the following integral formulas of Minkowski type for compact hypersurfaces in $\mathbb{R}^{n+1}$.

Theorem 4.1. ([6], [7]) Let $X: M \rightarrow \mathbb{R}^{n+1}$ be an $n$-dimensional compact hypersurface without boundary, $F: S^{n} \rightarrow \mathbb{R}^{+}$be a smooth function which satisfies (1), then we have the following integral formulas of Minkowski type:

$$
\int_{M}\left(H_{r}^{F} F \circ \nu+H_{r+1}^{F}\langle X, \nu\rangle\right) \mathrm{d} A=0, \quad r=0,1, \cdots, n-1 .
$$

Now, we let $X: M \rightarrow \mathbb{R}^{n+1}$ be a compact embedded hypersurface without boundary, then $M$ is a boundary of some compact domain $D \subset \mathbb{R}^{n+1}$, let $\nu$ be the unit inner normal vector field of $M$.

Lemma 4.1. For any fixed point $y \in D \backslash X(M)$, there exists at least a point $p \in M$ such that

$$
y-p=d_{F}(M, y) \phi \circ \nu(p) .
$$

Proof. From the compactness of $M$ and the continuity of the function $d_{F}$, there exists $p \in M$ such that $d_{F}(p, y)=\inf \left\{d_{F}(q, y) \mid q \in M\right\}$.

Let $Z: M \rightarrow S^{n}$ be defined by

$$
y-q=F^{*}(y-q) \phi \circ Z(q) .
$$


Then we have

$$
d_{F}(q, y)=\frac{\langle y-q, Z(q)\rangle}{F(Z(q))}
$$

Differentiating (26), we get

$$
\mathrm{d} d_{F}(q, y)=-\frac{\langle\mathrm{d} q, Z(q)\rangle}{F(Z(q))} .
$$

So, from the minimum of $p$, we get $\langle\mathrm{d} p, Z(p)\rangle=0$, thus $Z(p)= \pm \nu(p)$. If $Z(p)=-\nu(p)$, then $\langle\phi \circ Z(p), \nu(p)\rangle=-F(Z(p))<0$, so the line segment connecting $p$ and $y$ must intersect $X(M)$ at another point $\tilde{p}$, therefore $F^{*}(y-q)$ can not attain its minimum at $p$. Thus, $Z(p)=\nu(p)$ is the unit inner normal vector.

Lemma 4.2. Let $X: M \rightarrow \mathbb{R}^{2}$ be a simple closed curve and denote its arc parameter by $s$. Suppose $c(p)=1 / \lambda(p)$ for some point $p \in M$, then we must have $\lambda^{\prime}(p)=0$, where $\lambda$ is the anisotropic curvature and ' denote derivative with respect to the arc parameter.

Proof. Let $x_{0}=p+c(p) \phi \circ \nu(p)$, define $\tau: M \rightarrow \mathbb{R}$ by:

$$
\tau(q)=F^{*}\left(x_{0}-q\right),
$$

then there exists a function $W: M \rightarrow S^{1}$ such that

$$
x_{0}-q=\tau(q) \phi \circ W(q), \quad W(p)=\nu(p) .
$$

From the definition of $c(p)$, we have

$$
\tau(q) \geq c(p), \forall q \in M, \quad \tau(p)=c(p) .
$$

Differentiating (28), we get

$$
-T(q)=\tau^{\prime}(q) \phi \circ W(q)+\tau(q) a(W(q)) W^{\prime}(q),
$$

where $T$ denotes the tangent vector of $M, a=D^{2} F+F$.

By taking inner product with $W(q)$ in (30), we obtain

$$
F(W(q)) \tau^{\prime}(q)=-\langle T(q), W(q)\rangle .
$$

Thus, we have $\tau^{\prime}(p)=0$ by $W(p)=\nu(p)$. Then, from (30),

$$
W^{\prime}(p)=-T(p) /(a c(p))=-\lambda(p) T(p) / a=-k(p) T(p)=\nu^{\prime}(p),
$$

where $k$ is the curvature of $M$.

Differentiating (31), we get

$$
(F \circ W)^{\prime}(q) \tau^{\prime}(q)+F(W(q)) \tau^{\prime \prime}(q)=-\left\langle T^{\prime}(q), W(q)\right\rangle-\left\langle T(q), W^{\prime}(q)\right\rangle .
$$

Evaluating (33) at $q=p$, we get

$$
F(\nu(p)) \tau^{\prime \prime}(p)=-\langle k(p) \nu(p), \nu(p)\rangle-\langle T(p),-k(p) T(p)\rangle=0,
$$


so $\tau^{\prime \prime}(p)=0$. Thus, differentiating (33) and evaluating it at $p$, we obtain

$$
F(\nu(p)) \tau^{\prime \prime \prime}(p)=-\left\langle T^{\prime \prime}(p), \nu(p)\right\rangle-2\left\langle T^{\prime}(p), W^{\prime}(p)\right\rangle-\left\langle T(p), W^{\prime \prime}(p)\right\rangle .
$$

Differentiating (30) and evaluating at $p$, we get

$$
-T^{\prime}(p)=c(p)\left((a \circ W)^{\prime}(p) W^{\prime}(p)+a \circ \nu(p) W^{\prime \prime}(p)\right) .
$$

As $W^{\prime}(p)=\nu^{\prime}(p)$, so $(a \circ W)^{\prime}(p)=(a \circ \nu)^{\prime}(p)$, through a direct calculation, we have

$$
W^{\prime \prime}(p)=\frac{k(p)(a \circ \nu)^{\prime}(p)}{a \circ \nu(p)} T(p)-k^{2}(p) \nu(p) .
$$

From (32), (35) and $T^{\prime \prime}=-k^{2} T+k^{\prime} \nu$, we obtain

$$
F(\nu(p)) \tau^{\prime \prime \prime}(p)=-k^{\prime}(p)-\frac{k(p)(a \circ \nu)^{\prime}(p)}{a \circ \nu(p)}=-\frac{\lambda^{\prime}(p)}{a \circ \nu(p)} .
$$

As $\tau(q) \geq \tau(p)$ holds for all $q \in M$, and $\tau^{\prime}(p)=\tau^{\prime \prime}(p)=0$, so we must have $\tau^{\prime \prime \prime}(p)=0$, thus $\lambda^{\prime}(p)=0$ as expected.

Let $f: D \rightarrow \mathbb{R}$ be an integrable function. From Lemma 3.1, Lemma 3.4 and Lemma 4.1, we have the following formula of integration

$$
\int_{D} f d V=\int_{M} \int_{0}^{c(p)} f\left(\exp _{p}(t \phi(\nu(p)))\right) E(p, t) \mathrm{d} t \mathrm{~d} A,
$$

where $E(p, t)$ is given by

$$
d V\left(\exp _{p}(t \phi(\nu(p)))\right)=E(p, t) \mathrm{d} t \mathrm{~d} A .
$$

If $x$ denotes the position vector in $\mathbb{R}^{n+1}$, we have $\bar{\triangle}|x|^{2}=2(n+1)$, where $\bar{\triangle}$ is the Euclidean Laplacian. From the Stokes Theorem, we have

$$
-\int_{M}\langle X, \nu\rangle \mathrm{d} A=(n+1) V
$$

where $V$ the volume of $D$. From (11), we have

$$
d V(p+t \phi(\nu(p)))=\operatorname{det}\left(I-t S_{F}\right) F \circ \nu \mathrm{d} t \mathrm{~d} A=\left(1-t \lambda_{1}\right) \cdots\left(1-t \lambda_{n}\right) F \circ \nu \mathrm{d} t \mathrm{~d} A .
$$

Letting $f \equiv 1$ in (37) and taking into account that $E(p, t)=\left(1-t \lambda_{1}\right) \cdots\left(1-t \lambda_{n}\right) F \circ \nu$, we have

$$
V=\int_{M} \int_{0}^{c(p)}\left(1-t \lambda_{1}\right) \cdots\left(1-t \lambda_{n}\right) F \circ \nu \mathrm{d} t \mathrm{~d} A
$$

Theorem 4.2. Let $X: M \rightarrow \mathbb{R}^{n+1}$ be a compact hypersurface without boundary embedded in Euclidean space. If the anisotropic mean curvature $H_{1}^{F}$ of $X$ with respect to the unit inner normal $\nu$ is everywhere positive on $M$, then we have

$$
\int_{M} \frac{F \circ \nu}{H_{1}^{F}} \mathrm{~d} A \geq(n+1) V
$$


where $V$ is the volume of the compact domain determined by $M$. Moreover, the equality holds in (41) if and only if up to translations, $X(M)=\rho W_{F}$, where $\rho=-1 / H_{1}^{F}$ is a constant.

Proof. Firstly, if $X(M)=\rho W_{F}$, then $H_{1}^{F}=-1 / \rho=$ constant. So, by the integral equalities of Minkowski type (24) and (39), the equality in (41) holds.

For $p \in M$, by Lemma 3.3 , we have

$$
c(p) \leq 1 / \lambda_{\max } \leq 1 / H_{1}^{F}(p) .
$$

Moreover, if $t \in[0, c(p))$, we have

$$
\left(1-t \lambda_{1}\right) \cdots\left(1-t \lambda_{n}\right) \leq\left(1-t H_{1}^{F}\right)^{n},
$$

the equality holds only at points where $\lambda_{1}=\cdots=\lambda_{n}$. Thus, by putting (42), (43) into (40), we get

$$
V \leq \int_{M} \int_{0}^{1 / H_{1}^{F}}\left(1-t H_{1}^{F}\right)^{n} F \circ \nu \mathrm{d} t \mathrm{~d} A=\frac{1}{n+1} \int_{M} \frac{F \circ \nu}{H_{1}^{F}} \mathrm{~d} A,
$$

and the equality holds if and only if $\lambda_{1}=\cdots=\lambda_{n}=1 / c(p)$. Therefore, by Lemma 2.3, if $n \geq 2$ and the equality holds, then up to translations, $X(M)=\rho W_{F}$, where $\rho=-1 / H_{1}^{F}$. If $n=1$, then from Lemma 4.2, $\lambda=H_{1}^{F}$ is a constant, so from Lemma 2.2, up to translations, $X(M)=\rho W_{F}$, where $\rho=-1 / H_{1}^{F}$.

Remark 4.1. By Lemma 2.2, Theorem 1.3 is true for $n=1$ even without the assumption of embedding. So, in order to prove Theorem 1.3, we actually don't need to prove the case $n=1$ of Theorem 4.2. We prove it here only for completeness.

If $F \equiv 1$ in Theorem 4.2, then we obtain

Corollary 4.1. ([16], 21]) Let $X: M \rightarrow \mathbb{R}^{n+1}$ be a compact hypersurface without boundary embedded in Euclidean space. If the mean curvature $H$ of $X$ with respect to the unit inner normal $\nu$ is everywhere positive on $M$, then we have

$$
\int_{M} \frac{1}{H} \mathrm{~d} A \geq(n+1) V
$$

where $V$ is the volume of the compact domain determined by $M$. Moreover, the equality holds if and only if $X(M)$ is a round sphere.

\section{Proof of Theorem 1.3}

We divide into two cases:

Case 1. $\nu$ is the unit inner normal vector field. Since $M$ is compact without boundary, one can find a point where all the principal curvatures with respect to $\nu$ are positive. It follows from the positive definiteness of $A_{F}$ that all the anisotropic 
principal curvatures at this point with respect to $\nu$ are positive too. Thus $H_{r}^{F}$ is a positive constant. From Lemma 2.1, we have that $H_{1}^{F}, \cdots, H_{r-1}^{F}>0,\left(H_{r}^{F}\right)^{1 / r} \leq H_{1}^{F}$ and $H_{r-1}^{F} \geq\left(H_{r}^{F}\right)^{(r-1) / r}$. Using Theorem 4.2, we have

$$
(n+1)\left(H_{r}^{F}\right)^{1 / r} V \leq \int_{M} F \circ \nu \mathrm{d} A,
$$

and the equality holds if and only if up to translations, $X(M)=-\rho W_{F}$, where $\rho=$ $1 / H_{1}^{F}$ is a constant.

Since $H_{r}^{F}$ is a positive constant and $\left(H_{r}^{F}\right)^{1 / r} \leq H_{1}^{F}$, by Theorem 4.1, we have

$$
\begin{aligned}
0 & =\int_{M}\left(H_{r-1}^{F} F \circ \nu+H_{r}^{F}\langle X, \nu\rangle\right) \mathrm{d} A \geq \int_{M}\left(\left(H_{r}^{F}\right)^{(r-1) / r} F \circ \nu+H_{r}^{F}\langle X, \nu\rangle\right) \mathrm{d} A \\
& =\left(H_{r}^{F}\right)^{(r-1) / r} \int_{M}\left(F \circ \nu+\left(H_{r}^{F}\right)^{1 / r}\langle X, \nu\rangle\right) \mathrm{d} A .
\end{aligned}
$$

As $H_{r}^{F}$ is a positive constant, using (39) we have

$$
\int_{M} F \circ \nu \mathrm{d} A-(n+1)\left(H_{r}^{F}\right)^{1 / r} V=\int_{M}\left(F \circ \nu+\left(H_{r}^{F}\right)^{1 / r}\langle X, \nu\rangle\right) \mathrm{d} A \leq 0 .
$$

Hence, the equality in (44) holds, so up to translations, $X(M)=\rho W_{F}$, where $\rho=$ $-1 / H_{1}^{F}$ is a constant.

Case 2. $\nu$ is the unit outer normal vector field. The conclusion follows as in Case 1 by considering the function $\tilde{F}: S^{n} \rightarrow \mathbb{R}^{+}$defined by $\tilde{F}(x)=F(-x)$ instead of $F$. This completes the proof of Theorem 1.3 .

Acknowledgements The authors would like to thank Professor F. Morgan for his helpful suggestions and comments on the original version of this paper.

\section{REFERENCES}

1. A. D. Alexandrov, Uniqueness theorems for surfaces in the large I, Vestnik Leningrad Univ., 11(1956), 5-17.

2. J. E. Brothers and F. Morgan, The isoperimetric theorem for general integrands, Michigan Math. J., 41(1994), 419-431.

3. U. Clarenz, The Wulff-shape minimizes an anisotropic Willmore functional, Interfaces and Free Boundaries, 6(2004), 351-359.

4. L. F. Cao and H. Li, r-minimal submanifolds in space forms, Ann. Global Anal. Geom., 32(2007), 311-341.

5. L. Gårding, An inequality for hyperbolic polynomials, J. Math. Mech., 8(1959), 957-965.

6. Y. J. He and H. Li, Integral formula of Minkowski type and new characterization of the Wulff shape, arXiv:math.DG/0703187, 2007, to appear in Acta Math. Sinica.

7. Y. J. He and H. Li, A new variational characterization of the Wulff shape, Diff. Geom. App., (2007), doi:10.1016/j.difgeo.2007.11.030.

8. O. Hijazi, S. Montiel and Xiao Zhang, Dirac operator on embedded hypersurfaces, Math. Res. Lett., 8(2001), 195-208.

9. M. Koiso, Geometry of surfaces with constant anisotropic mean curvature, Symposium on the differential geometry of submanifolds, Valenciennes, July, 2007, 113-125. 
10. M. Koiso and B. Palmer, Geometry and stablity of surfaces with constant anisotropic mean curvature, Indiana Univ. Math. J., 54(2005), No.6, 1817-1852.

11. M. Koiso and B. Palmer, Stability of anisotropic capillary surfaces between two parallel planes, Calc. Var. Partial Differential Equations, 25(2006), 275-298.

12. M. Koiso and B. Palmer, Anisotropic capillary surfaces with wetting energy, Calc. Var. Partial Differential Equations, 29(2007), 295-345.

13. M. Koiso and B. Palmer, Uniqueness theorems for stable anisotropic capillary surfaces, Siam J. Math. Anal., 39(2007), 721-741.

14. H. Li, Hypersurfaces with constant scalar curvature in space forms, Math. Ann., 305(1996), 665672 .

15. F. Morgan, Planar Wulff shape is unique equilibrium, Proc. Amer. Math. Soc, 133(2004), 809-813.

16. S. Montiel and A. Ros, Compact hypersurfaces: The Alexandrov theorem for higher order mean curvatures, in Lawson, B. and Tenenblat, K. (eds), Differential Geometry, Pitman Monographs, Vol. 52, Longman, Essex, 1991, 279-296.

17. B. Palmer, Stability of the Wulff shape, Proc. Amer. Math. Soc., 126(1998), 3661-3667.

18. R. Reilly, The relative differential geometry of nonparametric hypersurfaces, Duke Math. J., 43(1976), 705-721.

19. R. Reilly, Applications of the Hessian operator in a Riemannian manifold, Indiana Univ. Math. J., 26(1977), 459-472.

20. A. Ros, Compact hypersurfaces with constant scalar curvature and a congruence theorem, J. Diff. Geom., 27(1988), 215-220.

21. A. Ros, Compact hypersurfaces with constant higher order mean curvatures, Revista Mathmática Iberoamericana, 3(1987), 447-453.

22. J. Taylor, Crystalline variational problems, Bull. Amer. Math. Soc., 84(1978), 568-588.

23. S. Winklmann, A note on the stability of the Wulff shape, Arch. Math., 87(2006), 272-279.

24. S.-T. Yau, Problem section, Seminar on Differential Geometry, Annals Math. Studies, No. 102, Princeton University Press, Princeton, NJ, 1982.

School of Mathematical Sciences, Shanxi University, Taiyuan 030006, P. R. China.

E-mail address: heyijun@sxu.edu.cn

Department of Mathematical Sciences, Tsinghua University, Beijing 100084, P. R. China.

E-mail address: hli@math.tsinghua.edu.cn

Department of Mathematical Sciences, Tsinghua University, Beijing 100084, P. R. China.

E-mail address: hma@math.tsinghua.edu.cn

Department of Mathematical Sciences, Tsinghua University, Beijing 100084, P. R. China.

E-mail address: gejq04@mails.tsinghua.edu.cn 